\title{
Primary Retroperitoneal Müllerian Adenocarcinoma: A Case Report and Literature Review
}

\author{
C. Spinelli $i^{\mathrm{a}}$ \\ S. Strambia \\ D. Tartaglia ${ }^{a}$ \\ G. Di Franco ${ }^{a}$ \\ V. Pucci ${ }^{a}$ \\ P. Favianab \\ M. Lencioni ${ }^{\mathrm{c}}$
}

${ }^{a}$ Department of Surgical, Clinical, Molecular Pathology and Critical Area, UO Chirurgia Generale 2, ${ }^{b}$ Department of Surgical, Clinical, Molecular Pathology and Critical Area, UO Anatomia Patologica 3, and ${ }^{C}$ Department of Oncology, Transplants, and New Technologies, UO Oncologia 1, University of Pisa, Pisa, Italy

\section{Key Words}

Malignant mixed müllerian tumor · Primary retroperitoneal müllerian adenocarcinoma . Gynecological malignancy

\section{Abstract}

Primary retroperitoneal müllerian adenocarcinoma (PRMA) is an extremely rare clinical entity. We report the case of a 54-year-old woman who presented with a mass in the right lower retroperitoneum, identified during an ultrasound exam. Computed tomography confirmed a retroperitoneal mass measuring $11 \mathrm{~cm}$. The patient underwent laparotomy and the mass was completely excised. The histopathological exam revealed PRMA.

(C) 2013 S. Karger AG, Basel

\section{Introduction}

A malignant mixed müllerian tumor (MMMT) that develops from the female genital tract is a rare clinical entity, accounting for less than $1 \%$ of all gynecological malignancies [1]. Since müllerian ducts develop to form the fallopian tubes, uterus and the upper third of the vagina, MMMTs are usually found in these structures or, sometimes, in the ovary.

There are also extremely rare MMMTs of extragenital origin: they can arise from the peritoneum or be retroperitoneal [2-5]. To the best of our knowledge, primary retroperitoneal müllerian adenocarcinoma (PRMA) has been reported in only 6 cases in the literature to date (table 1). This tumor often presents in elderly menopausal women and is highly

Prof. Claudio Spinelli

Department of Surgical, Clinical, Molecular Pathology and Critical Area

University of Pisa, Via Paradisa 2

IT-56124 Pisa (Italy)

E-Mail c.spinelli@dc.med.unipi.it 
aggressive. Its growth pattern and size are variable, and histological evaluation shows both carcinoma (epithelial) and sarcoma (mesenchymal) components; thus, the disorder is also called 'carcinosarcoma'. Here, we report the case of PRMA in a 54-year-old woman, underlining its clinical, radiological and surgical features, and a review of the literature.

\section{Case Report}

A 54-year-old primiparous woman presented with a 6-month history of mild abdominal pain and distension of the right portion of the abdomen. Her family history was not contributory. Her past surgical history included a total thyroidectomy for papillary carcinoma followed by radioiodine therapy 3 years before.

Physical examination revealed a slightly tender, ill-defined mass over the left abdomen. The remaining systemic examination did not reveal any coexistent lesion. Laboratory tests, including complete blood count, chemistry profile, urinalysis and chest X-ray, were all within normal limits. Serum tumor markers were negative.

An abdominal ultrasound (US) documented the presence of a coarse, partially cystic mass in the right retroperitoneal region. Abdominopelvic CT and MRI revealed a large unilocular cystic mass measuring $11 \mathrm{~cm}$ with an enhancing solid portion and partial water content, located in the retroperitoneal space adjacent to the lower pole of the right kidney, with wall calcifications (fig. 1). There was no evidence of extracystic extension or distant metastasis. On suspicion of a hydatid cyst, serological tests were performed and yielded negative results. A gynecological examination, including hysteroscopy and Pap smear, was also performed. It was negative for utero-adnexal pathology.

The patient underwent laparotomy with subcostal transverse incision. On opening the peritoneal cavity, ascitic fluid was found and sampled. Cytological examination of the fluid did not reveal the presence of malignant cells. Abdominal cavity exploration revealed no abnormal findings in the liver, stomach, large bowel and spleen, and the uterus, ovaries, fallopian tubes and appendix were all normal. Sequential exploration of the small intestine revealed the presence of an exophytic lesion about $1 \mathrm{~cm}$ in diameter, which was removed and whose definitive histological examination was compatible with fibroma of the small intestine. A large, encapsulated cystic mass was found in the right retroperitoneum, below the lower pole of the right kidney that was displaced posteriorly and cranially to the mass. The cecum, right colon and right colic flexure were displaced medially to the mass. The mass was dissected from the surrounding structures. A fragment of the cystic wall was sent for extemporaneous histological examination and it was compatible with a fibrous wall denuded of lining epithelium. Complete excision of the cyst was performed. The postoperative period was uneventful, and the patient was discharged home 5 days after surgery.

The gross pathological findings showed a $12 \times 10-\mathrm{cm}$ cystic mass with a smooth surface. The cystic wall measured $0.3 \mathrm{~cm}$ at maximum thickness. The tumor was composed of malignant epithelial and stromal components. Microscopically, the fibrous wall of the cyst delimited an extensively necrotic lesion containing chronic inflammation, calcification, cholesterol crystals and hemorrhagic extravasation. On hematoxylin and eosin staining, the entire tumor showed cellular whorls dispersed amidst pale sarcomatous stroma. The cells in whorls were round to short, spindle shaped and had moderate mitotic activity. The sections, however, revealed whorled glandular areas.

A panel of immunohistochemical tests was performed: whorled glandular areas were positive for cytokeratin (CK19) and negative for vimentin, confirming their epithelial nature; they were also negative for CK20 and calretinin. The stromal component of the tumor, 
highlighted on vimentin stain (vimentin), was positive for CD10. No immunoreactivity to estrogen and progesterone receptors was found. The presence of necrosis, cytonuclear atypia and immunophenotypic features were compatible with PRMA (fig. 2).

Six courses of adjuvant chemotherapy were administered with carboplatin at a dose of 5 AUC and taxol $175 \mathrm{mg} / \mathrm{mq} / \mathrm{ev}$. A follow-up abdominal CT, performed 9 months later, was negative for tumor recurrence. Written informed consent was obtained from the patient for publication of this case report and all accompanying images.

\section{Discussion}

Extragenital MMMTs occur infrequently. Patients usually present with specific clinical symptoms including abdominal distension, pain and/or a palpable mass [6]. The exact pathogenesis of PRMA is still unknown: Elnemr et al. [2] hypothesized that this tumor arises from residual hormone-independent cells of Müller ducts in the retroperitoneum, based on correlations with endometriosis and irradiation $[5,7,8]$.

In our patient we found no evidence which could be linked to endometrial implants or even a history of abdominal or pelvic irradiation, except for a radioactive iodine treatment.

The preoperative diagnostic management includes US, CT and, if necessary, MRI [3]. Preoperative diagnosis is rarely suspected because of the nonspecific symptoms and the scarce aid of imaging examinations. Although US, CT and MRI clearly detect cystic masses in ovarian or pelvic organs, diagnosis of a retroperitoneal tumor is extremely difficult [6]. When radiological exams do not reveal evidence of metastatic disease, patients must undergo laparotomy with the aim of eradicating the tumor completely. Due to its strong aggressiveness, it is recommended not to break the tumor mass during surgical eradication maneuvers, in order to avoid any peritoneal implantation $[2,9,10]$.

It was considered that the biological behavior of PRMA resembled that of epithelial ovarian cancer, so patients have been treated in a similar way, using the same regimen of adjuvant chemotherapy for ovarian tumors; the role of radiotherapy remains controversial $[1,11,12]$. As shown in the literature, because of the very small number of cases thus far presented, no evidence-based management guidelines are available.

Further studies will be needed to determine the correct therapeutic strategy to follow, evaluating long-term follow-up, course and prognosis of this disease.

\section{Disclosure Statement}

The authors declare that they have no competing interests.

\section{References}

1 Huang CC, Ma CG, Huang WT, et al: Primary malignant mixed Müllerian tumor arising from the mesorectum with a synchronous ovarian cancer: a case report and review of the literature. J Med Case Rep 2011;5:15.

-2 Elnemr A, Yonemura Y, Shinbo M, Nishino E, et al: Primary retroperitoneal mullerian adenocarcinoma. Rare Tumors 2010;2:e6.

-3 Yang DM, Jung DH, Kim H, et al: Retroperitoneal cystic masses: CT, clinical, and pathologic findings and literature review. Radiographics 2004;24:1353-1365.

4 Berretta R, Patrelli TS, Faioli R, et al: Secondary müllerian system: an atypical case of tumor originating from vestigial müllerian cells embedded in the peritoneum. Clin Genitourin Cancer 2013;11:365-369.

5 Kanis M, Kesterson JP, Shroff S, et al: Malignant mixed müllerian tumor of primary peritoneal origin. Ann Diagn Pathol 2011;15:273-277. 
Spinelli et al.: Primary Retroperitoneal Müllerian Adenocarcinoma: A Case Report and Literature Review

6 de León DC, Pérez-Montiel D, Chanona-Vilchis J, et al: Primary retroperitoneal mucinous cystadenocarcinoma: report of two cases. World J Surg Oncol 2007;5:5.

7 Booth C, Zahn C, McBroom J, et al: Retroperitoneal Mullerian carcinosarcoma associated with endometriosis: a case report. Gynecol Oncol 2004;93:546-549.

-8 Ferrie RK, Ross RC: Retroperitoneal Mullerian carcinosarcoma. Can Med Assoc J 1967;97:1290-1292.

-9 Iura A, Sasajima Y, Katsumata N, et al: Serous adenocarcinoma of the retroperitoneum, as a type of multifocal müllerian carcinoma. Int J Clin Oncol 2009;14:254-257.

10 Kassab A, El-Bialy G, Clark J, et al: Unusual presentation of 22-kilogram retroperitoneal müllerian serous cystadenoma. Gynecol Oncol 2007;104:257-259.

$\checkmark 11$ Mok JE, KimYM, Jung MH, et al: Malignant mixed mullerian tumors of the ovary: experience with cytoreductive surgery and platinum-based combination chemotherapy. Int J Gynecol Cancer 2006;16:101105.

12 Yonemura Y, Endou Y, Shinbo M, et al: Safety and efficacy of bidirectional chemotherapy for treatment of patients with peritoneal dissemination from gastric cancer: selection for cytoreductive surgery. J Surg Oncol 2009;100:311-316.

Table 1. Reports of primary retroperitoneal and peritoneal müllerian adenocarcinomas

\begin{tabular}{llllllll}
\hline Study (first author) & $\begin{array}{l}\text { Age, } \\
\text { years }\end{array}$ & Site & Size, $\mathrm{cm}$ & Growth pattern & $\begin{array}{l}\text { Uterine adnexal } \\
\text { lesion }\end{array}$ & $\begin{array}{l}\text { Adjuvant } \\
\text { therapy }\end{array}$ & Outcome \\
\hline Ferrie, 1967 [8] & 47 & Retroperitoneal & 11 & Cystic & $\begin{array}{l}\text { Previous } \\
\text { hydatidiform mole }\end{array}$ & NS & NS \\
\hline Booth, 2004 [7] & 71 & Retroperitoneal space & $9 \times 7 \times 6.5$ & Solid and cystic & $\begin{array}{l}\text { Uterine } \\
\text { leiomyomata }\end{array}$ & Pelvic RT & NED 32 months \\
\hline de León, 2007 [6] & 36 & Retroperitoneal space & $19 \times 16 \times 12$ & Solid and cystic & None & CT & AWD 9 months \\
\hline de León, 2007 [6] & 21 & Retroperitoneal space & $26 \times 18 \times 16$ & Solid and cystic & None & Patient & NED 6 months \\
\hline Iura, 2009 [9] & 66 & Retroperitoneal space & $20 \times 9.5 \times 8.5$ & Solid and cystic & $\begin{array}{l}\text { Serous } \\
\text { adenocarcinoma }\end{array}$ & CT & $\begin{array}{l}\text { AWD 32 } \\
\text { months }\end{array}$ \\
\hline Elnemer, 2010 [2] & 46 & Retroperitoneal space & $15 \times 10$ & Cystic & None & CT & AWD 6 months \\
\hline Kanis, 2011 [5] & 80 & Peritoneal & $15 \times 10$ & Solid & None & CT & DOD 4 months \\
\hline Kanis, 2011 [5] & 57 & Peritoneal & $15 \times 20 \times 15$ & Solid and cystic & None & CT & NED 4 months \\
\hline Berretta, 2013 [4] & 72 & Peritoneal & $33 \times 33 \times 11$ & Solid and cystic & None & NS & NED 12 months \\
\hline Present report, 2013 & 54 & Retroperitoneal space & $11 \times 10$ & Cystic & None & CT & NED 9 months \\
\hline
\end{tabular}

$\mathrm{AWD}=$ Alive with disease; $\mathrm{CT}$ = chemotherapy; $\mathrm{DOD}$ = died of disease; NED = no evidence of disease; $\mathrm{NS}$ = not specified; RT = radiation therapy. 
Spinelli et al:: Primary Retroperitoneal Müllerian Adenocarcinoma: A Case Report and Literature Review
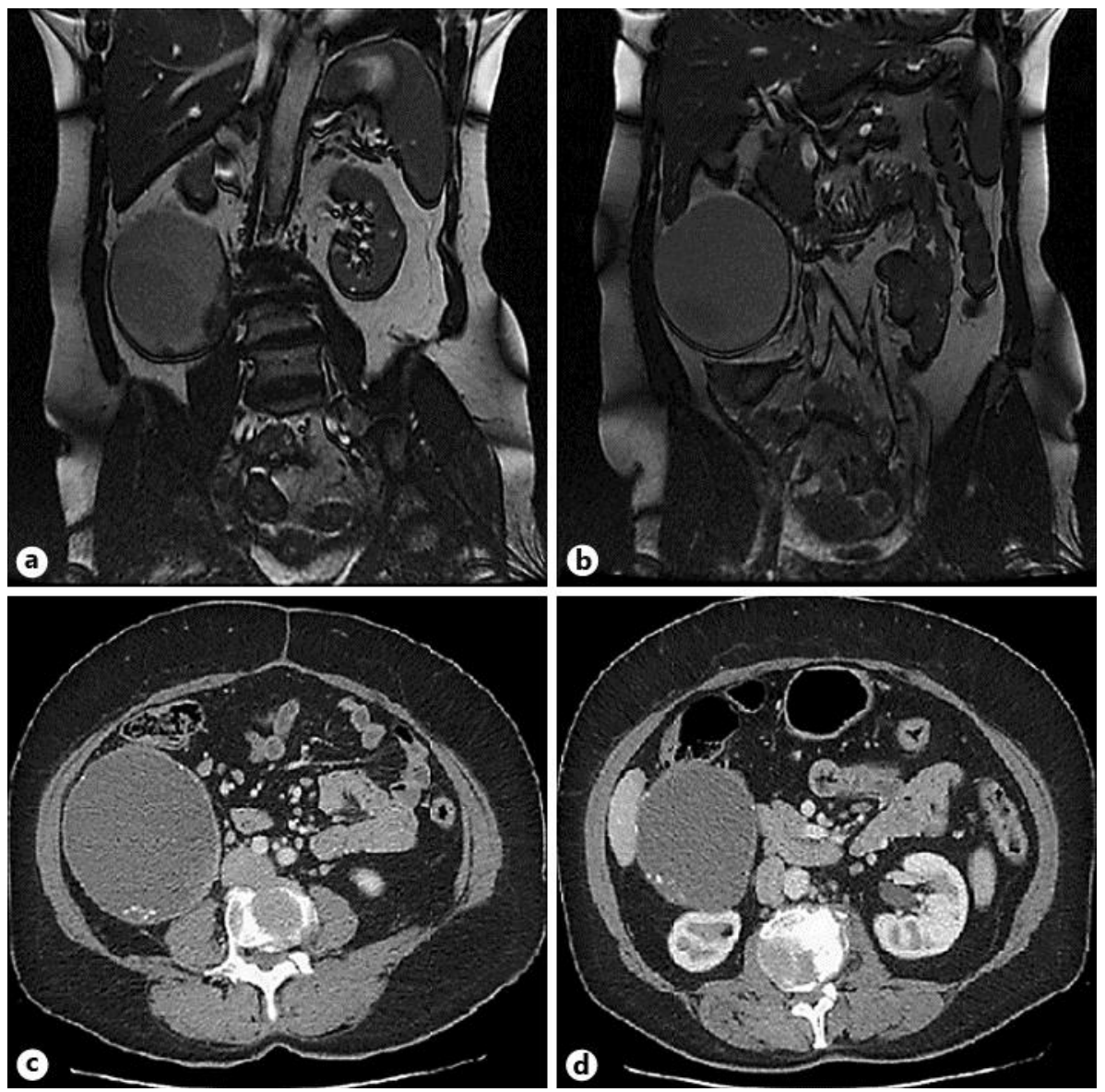

Fig. 1. MRI (a, b) and abdominopelvic CT (c, d) clearly show a large unilocular cystic mass in the right retroperitoneal space. 


\section{Case Reports in Oncology}

\begin{tabular}{l|l}
\hline Case Rep Oncol 2013;6:616-621 & \\
\hline DOI: 10.1159/000357424 & $\begin{array}{l}\text { ○ 2013 S. Karger AG, Basel } \\
\text { www.karger.com/cro }\end{array}$ \\
\hline
\end{tabular}

Spinelli et al.: Primary Retroperitoneal Müllerian Adenocarcinoma: A Case Report and Literature Review

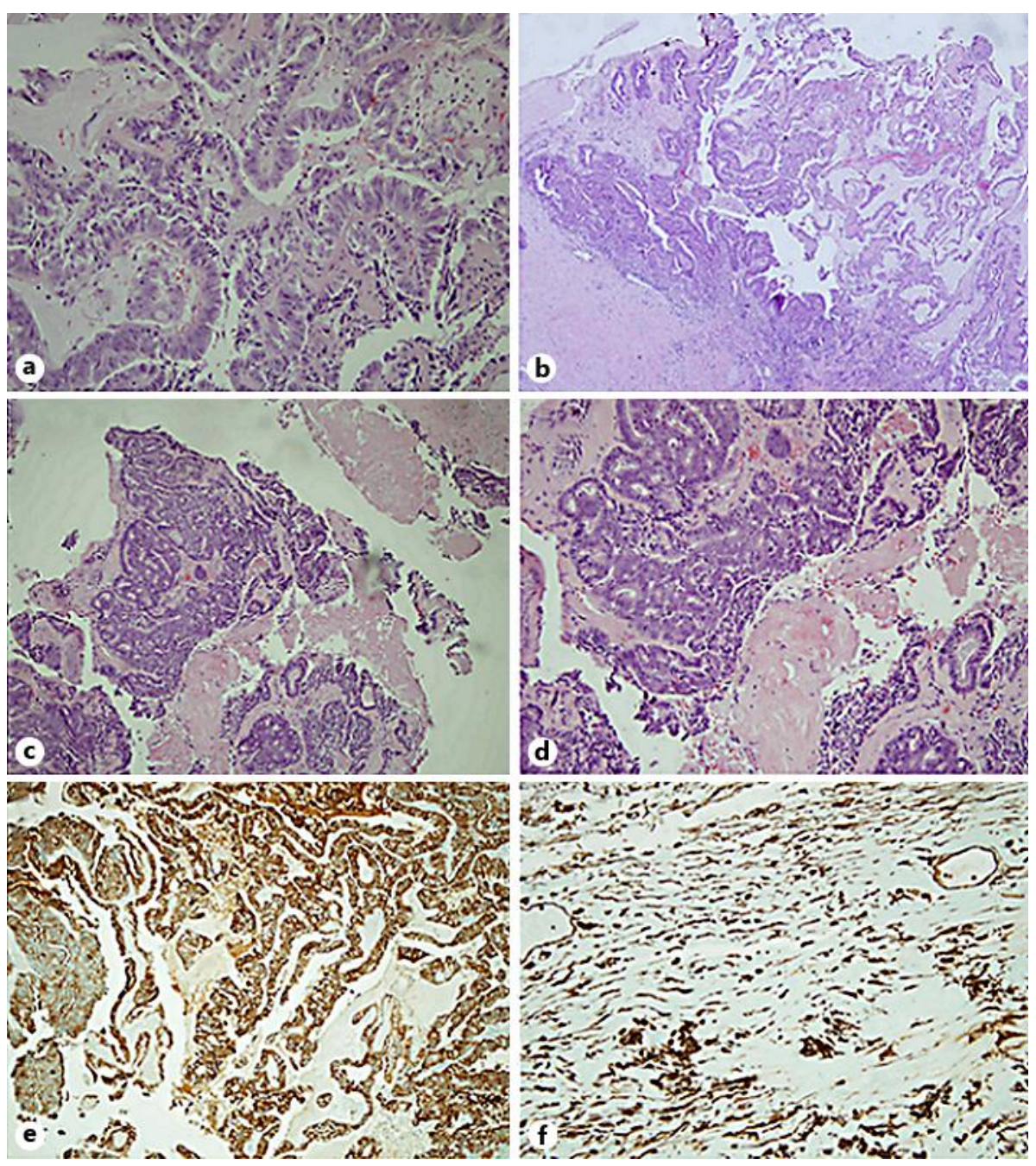

Fig. 2. Histopathological findings. MMMT, also known as carcinosarcoma, is composed of malignant epithelial and stromal components. Glandular epithelial (a-e) and sarcomatous (f) elements of the lesion. On hematoxylin and eosin (H\&E) staining, the whole mass shows cellular whorls dispersed amidst pale sarcomatous stroma. a H\&E. $\times 40$. b H\&E. $\times 20$. c H\&E. $\times 20$. d H\&E. $\times 40$. e Positivity for cytokeratin (CK19) of whorled glandular areas confirms their epithelial nature. $f$ Vimentin reveals the sarcomatous nature of the stromal component. 